Georgian Mathematical Journal

Volume 12 (2005), Number 4, 671-678

\title{
ON MUTUALLY COHERENT ENUMERATIONS OF COUNTABLE SETS
}

\author{
ALEXANDER KHARAZISHVILI
}

\begin{abstract}
The notion of compatibility of enumerations of countable (in particular, finite) sets is introduced and investigated. Some purely combinatorial problems connected with this notion are considered and several conditions are presented for the existence of mutually coherent enumerations of all members of a given family of countable sets.
\end{abstract}

2000 Mathematics Subject Classification: 03E05, 03E20, 05A05, 05A99.

Key words and phrases: Mutually coherent enumerations, Latin square, initial partial selector, point-finite family of sets, admissible family of sets.

Let $\omega$ denote the set of all natural numbers and let $A$ be an arbitrary countable set. There exists a bijection $\theta: K \rightarrow A$ where $K$ is an initial subinterval of the set $\omega \backslash\{0\}=\{1,2, \ldots, n, \ldots\}$ If $A$ is infinite, then $K=\omega \backslash\{0\}=$ $\{1,2, \ldots, n, \ldots\}$. If $A$ is finite, then $K$ is of the form $K=\{1,2, \ldots, k\}$ for some $k \in \omega$. In both these cases we can write

$$
A=\left\{a_{1}, a_{2}, \ldots, a_{n}, \ldots\right\}
$$

where $a_{n}=\theta(n)$ for any $n \in K$, and we can say that a certain enumeration of $A$ is determined by the mapping $\theta$.

Let $B$ be another countable set and let

$$
B=\left\{b_{1}, b_{2}, \ldots, b_{n}, \ldots\right\}
$$

be an enumeration of $B$. We say that these two enumerations are coherent (or compatible) if the relation $a_{n}=b_{m}$ implies $n=m$.

Accordingly, we say that a family $\left\{A_{i}: i \in I\right\}$ of countable sets admits mutually coherent enumerations of all its members if there exists a family

$$
\theta_{i}: K_{i} \rightarrow A_{i}(i \in I)
$$

of pairwise coherent enumerations for $\left\{A_{i}: i \in I\right\}$.

It is easy to see that the following two assertions are equivalent:

1) a family of countable sets $\left\{A_{i}: i \in I\right\}$ admits mutually coherent enumerations of all its members;

2) there exists a function

$$
f: \cup\left\{A_{i}: i \in I\right\} \rightarrow \omega \backslash\{0\}
$$

such that, for any index $i \in I$, the restriction $f \mid A_{i}$ is a bijection between the set $A_{i}$ and some initial subinterval of $\omega \backslash\{0\}$. 
Thus, the relation 2) can be regarded as another definition of mutually coherent (compatible) enumerations. In order to illustrate the notion introduced above, let us recall a well-known example from finite combinatorics.

Example 1. Fix a natural number $n \geq 1$ and consider a finite set

$$
Z=\left\{x_{\alpha, \beta}: \alpha \in\{1,2, \ldots, n\}, \beta \in\{1,2, \ldots, n\}\right\} .
$$

Denote also

$$
\begin{gathered}
X_{\alpha}=\left\{x_{\alpha, \beta}: \beta \in\{1,2, \ldots, n\}\right\}(\alpha=1,2, \ldots, n), \\
Y_{\beta}=\left\{x_{\alpha, \beta}: \alpha \in\{1,2, \ldots, n\}\right\}(\beta=1,2, \ldots, n), \\
\left\{A_{i}: i \in I\right\}=\left\{X_{\alpha}: \alpha \in\{1,2, \ldots, n\}\right\} \cup\left\{Y_{\beta}: \beta \in\{1,2, \ldots, n\}\right\} .
\end{gathered}
$$

In this case, the existence of mutually coherent enumerations for the members of $\left\{A_{i}: i \in I\right\}$ is equivalent to the existence of a Latin square with $n$ rows and $n$ columns. Since such Latin squares do exist for any natural $n \geq 1$, we claim that there are mutually coherent enumerations of the members of $\left\{A_{i}: i \in I\right\}$. Moreover, as is known, if $m$ is a natural number and $m<n$, then every Latin $(n \times m)$-rectangle can be extended to a Latin $(n \times n)$-square and, in general, there are various possibilities of appropriate extensions. Note that Latin $(n \times m)$-rectangles are closely connected with the Hall combinatorial theorem on pairwise distinct representatives. Detailed information about this topic and related problems of finite combinatorics can be found, e.g., in [1] or $[2]$.

Let $\left\{A_{i}: i \in I\right\}$ be a family of countable sets. It is reasonable to ask: what are necessary and sufficient conditions under which the family $\left\{A_{i}: i \in I\right\}$ admits mutually coherent enumerations of all its members?

In order to discuss this question, we should first introduce some simple preliminary notions.

Let $\left\{Z_{n}: n \in \omega \backslash\{0\}\right\}$ be a countable disjoint family of sets. We recall that a set $X$ is a partial selector of this family if the following relations hold:

a) $X \subset \cup\left\{Z_{n}: n \in \omega \backslash\{0\}\right\}$;

b) $\operatorname{card}\left(Z_{n} \cap X\right) \leq 1$ for each $n \in \omega \backslash\{0\}$.

We say that a set $X$ is an initial partial selector of $\left\{Z_{n}: n \in \omega \backslash\{0\}\right\}$ if $X$ satisfies the relations a), b) and the relation

c) for any natural number $n \geq 1$, if $X \cap Z_{n}=\varnothing$, then $(\forall m>n)\left(X \cap Z_{m}=\varnothing\right)$.

Now, the existence of mutually coherent enumerations can be formulated in terms of initial partial selectors.

Theorem 1. Let $\left\{X_{i}: i \in I\right\}$ be a family of countable sets. The following two assertions are equivalent:

(1) $\left\{X_{i}: i \in I\right\}$ admits mutually coherent enumerations of all its members;

(2) there exists a disjoint countable family of sets $\left\{Z_{n}: n \in \omega \backslash\{0\}\right\}$ such that every set $X_{i}(i \in I)$ is an initial partial selector of $\left\{Z_{n}: n \in \omega \backslash\{0\}\right\}$.

Proof. Suppose that (1) is valid and let

$$
f: \cup\left\{X_{i}: i \in I\right\} \rightarrow \omega \backslash\{0\}
$$


be a function which induces mutually coherent enumerations of all members of $\left\{X_{i}: i \in I\right\}$. For any natural number $n \geq 1$, let us define

$$
Z_{n}=\left\{x \in \cup\left\{X_{i}: i \in I\right\}: f(x)=n\right\}
$$

and consider the disjoint countable family of sets $\left\{Z_{n}: n \in \omega \backslash\{0\}\right\}$. Since for each index $i \in I$ the restriction $f \mid X_{i}$ is a bijection between $X_{i}$ and some initial subinterval of $\omega \backslash\{0\}$, we easily infer that each $X_{i}$ is an initial partial selector of $\left\{Z_{n}: n \in \omega \backslash\{0\}\right\}$, which shows that (2) is satisfied.

Conversely, suppose that (2) is valid and take a disjoint countable family of sets $\left\{Z_{n}: n \in \omega \backslash\{0\}\right\}$ as in (2). Further, define a function

$$
f: \cup\left\{Z_{n}: n \in \omega \backslash\{0\}\right\} \rightarrow \omega \backslash\{0\}
$$

by putting

$$
\operatorname{ran}\left(f \mid Z_{n}\right)=\{n\}\left(n \in \omega \backslash\{0\}, Z_{n} \neq \varnothing\right) .
$$

Since we have the inclusion

$$
\cup\left\{X_{i}: i \in I\right\} \subset \cup\left\{Z_{n}: n \in \omega \backslash\{0\}\right\},
$$

the function $f$ is also defined on the set $\cup\left\{X_{i}: i \in I\right\}$. Let us verify that $f$ induces mutually coherent enumerations for $\left\{X_{i}: i \in I\right\}$.

Fix an index $i \in I$ and consider the set $X_{i}$. If $X_{i}=\varnothing$, then there is nothing to prove. So suppose that $X_{i} \neq \varnothing$. Since $X_{i} \subset \cup\left\{Z_{n}: n \in \omega \backslash\{0\}\right\}$, there are natural numbers $n \geq 1$ for which $X_{i} \cap Z_{n} \neq \varnothing$. Only two cases are possible.

I. For any natural number $n \geq 1$, we have $X_{i} \cap Z_{n} \neq \varnothing$. In this case, keeping in mind that $X_{i}$ is a partial selector of $\left\{Z_{n}: n \in \omega \backslash\{0\}\right\}$, we deduce that $f \mid X_{i}$ is a bijection between $X_{i}$ and $\omega \backslash\{0\}$.

II. There exists a natural number $m$ such that $X_{i} \cap Z_{m+1}=\varnothing$. We may assume without loss of generality that $m$ is the smallest number with this property. Taking into account that $X_{i}$ is an initial partial selector of $\left\{Z_{n}: n \in \omega \backslash\{0\}\right\}$, we claim that $f \mid X_{i}$ is a bijection between the sets $X_{i}$ and $\{1,2, \ldots, m\}$. Thus, relation (1) is valid and the proof is completed.

However, in some sense Theorem 1 does not look satisfactory because the relation (2) of this theorem is not inner for the combinatorial structure of a given family of sets $\left\{X_{i}: i \in I\right\}$. Of course, we would like to have necessary and sufficient conditions for the existence of mutually coherent enumerations in terms of purely combinatorial properties of $\left\{X_{i}: i \in I\right\}$ (e.g., similarly to the above-mentioned Hall theorem on representatives). For the time being, such necessary and sufficient conditions are unknown. In this paper we are going to give some sufficient conditions for the existence of mutually coherent enumerations of all members of $\left\{X_{i}: i \in I\right\}$. First, let us consider the case where

$$
(\forall i \in I)\left(\operatorname{card}\left(X_{i}\right)<\omega\right) .
$$

One can expect that in this case a compactness type argument should work for establishing the existence of mutually coherent enumerations. Indeed, we are able to prove the following statement. 
Theorem 2. Let $\left\{X_{i}: i \in I\right\}$ be a family of finite sets and suppose that, for each finite subset $J$ of $I$, the members of the subfamily $\left\{X_{i}: i \in J\right\}$ admit mutually coherent enumerations. Then there are mutually coherent enumerations for the members of $\left\{X_{i}: i \in I\right\}$.

Proof. In fact, we will use a fairly standard argument based on the Tychonoff product theorem from general topology (see, e.g., [3]). Namely, first of all we equip the set

$$
\omega \backslash\{0\}=\{1,2, \ldots, n, \ldots\}
$$

with the discrete topology. In this manner we get a locally compact topological space which admits the Alexandroff compactification by adding a single point. The obtained compact space can be identified with the space

$$
\omega=\{0\} \cup\{1,2, \ldots, n, \ldots\}
$$

where the singleton $\{0\}$ has a local base consisting of all sets of the form

$$
\{0\} \cup\{n, n+1, n+2, \ldots\}(n \in \omega) .
$$

Actually, in our case $\omega$ is homeomorphic to the countable compact subset $\{0,1,1 / 2,1 / 3, \ldots, 1 / n, \ldots\}$ of the real line $R$.

Let us denote

$$
X=\cup\left\{X_{i}: i \in I\right\}
$$

and let

$$
E=\omega^{X}=\{f: f \text { is a mapping from } X \text { into } \omega\}
$$

By virtue of the above-mentioned Tychonoff product theorem, the space $E$ is compact. Now, for any subset $J$ of $I$, let us put:

$F_{J}=\{f \in E: f$ induces mutually coherent enumerations for the partial family $\left.\left\{X_{i}: i \in J\right\}\right\}$.

According to the assumption of the theorem, we have

$$
F_{J} \neq \varnothing
$$

whenever $J \subset I$ and $J$ is finite. Further, if $J_{1}$ and $J_{2}$ are any two subsets of $I$, then

$$
F_{J_{1} \cup J_{2}} \subset F_{J_{1}} \cap F_{J_{2}},
$$

which shows, in particular, that the family of sets

$$
\left\{F_{J}: J \subset I, \operatorname{card}(J)<\omega\right\}
$$

is centered in $E$. Let us verify that each member of this family is closed in $E$. For this purpose, fix a finite set

$$
J=\left\{i_{1}, i_{2}, \ldots, i_{m}\right\} \subset I
$$

and denote

$$
F_{i}=\left\{f \in E: f \mid X_{i} \text { is a bijection from } X_{i} \text { onto }\{1,2, \ldots, n(i)\}\right\}
$$

where $i \in J$ and $n(i)=\operatorname{card}\left(X_{i}\right)$. Obviously, we have the equality

$$
F_{J}=F_{i_{1}} \cap F_{i_{2}} \cap \cdots \cap F_{i_{m}} .
$$


Therefore, it suffices to establish the closedness of all sets $F_{i_{1}}, F_{i_{2}}, \ldots, F_{i_{m}}$. Let us fix $i \in J$ and represent the set $X_{i}$ in the form

$$
X_{i}=\left\{x_{1}, x_{2}, \ldots, x_{n(i)}\right\} .
$$

Put $k=(n(i))$ !. Denote also by $\left\{\phi_{1}, \phi_{2}, \ldots, \phi_{k}\right\}$ an injective family of all bijections acting from $\left\{x_{1}, x_{2}, \ldots, x_{n(i)}\right\}$ onto $\{1,2, \ldots, n(i)\}$. Finally, define

$$
F_{i, \phi_{r}}=\left\{f \in E: f \mid X_{i}=\phi_{r}\right\}(r \in\{1,2, \ldots, k\}) .
$$

Clearly, every set $F_{i, \phi_{r}}$ is closed in $E$. Taking into account the equality

$$
F_{i}=F_{i, \phi_{1}} \cup F_{i, \phi_{2}} \cup \cdots \cup F_{i, \phi_{k}},
$$

we claim that $F_{i}$ is closed for each $i \in J$ and, consequently, the set $F_{J}$ is closed, too. Thus, we have the centered family

$$
\left\{F_{J}: J \subset I, \operatorname{card}(J)<\omega\right\}
$$

of closed subsets of our compact space $E$, which yields

$$
\cap\left\{F_{J}: J \subset I, \operatorname{card}(J)<\omega\right\} \neq \varnothing .
$$

Take any $f \in \cap\left\{F_{J}: J \subset I, \operatorname{card}(J)<\omega\right\}$. It directly follows from the definition of $f$ that $f$ induces mutually coherent enumerations of all members of the given family $\left\{X_{i}: i \in I\right\}$. This ends the proof of Theorem 2 .

Remark 1. It is useful to compare the theorem just proved with the wellknown Rado lemma [4] which has numerous applications in mathematical logic, model theory and set theory (see, for instance, [5] and [6]). Note that the proof of a certain version of the Rado lemma (and the proof of the Hall combinatorial theorem for the case of an infinite family of finite sets) can also be carried out by using a similar argument based on the same Tychonoff product theorem (cf. [1] and [7]).

Now, let us turn our attention to the case where a family of sets $\left\{X_{i}: i \in I\right\}$ is countable and

$$
(\forall i \in I)\left(\operatorname{card}\left(X_{i}\right)=\omega\right) .
$$

On this occasion, we will formulate some natural sufficient conditions for the existence of mutually coherent enumerations of the members of $\left\{X_{i}: i \in I\right\}$.

We recall that a family $\left\{X_{i}: i \in I\right\}$ of nonempty sets is point-finite if

$$
\operatorname{card}\left(\left\{i \in I: x \in X_{i}\right\}\right)<\omega
$$

for every element $x \in \cup\left\{X_{i}: i \in I\right\}$. Note that in such a case we have the inequality

$$
\operatorname{card}(I) \leq \operatorname{card}\left(\cup\left\{X_{i}: i \in I\right\}\right)+\omega .
$$

We say that a family $\left\{X_{i}: i \in I\right\}$ is admissible if no set $X_{i_{0}}\left(i_{0} \in I\right)$ can be covered by finitely many members of $\left\{X_{i}: i \in I \backslash\left\{i_{0}\right\}\right\}$. 
Remark 2. Suppose that $\left\{X_{i}: i \in I\right\}$ is a point-finite family of sets satisfying the relation

$$
\operatorname{card}\left(\left\{i \in I: x \in X_{i}\right\}\right) \geq 2
$$

for each element $x \in \cup\left\{X_{i}: i \in I\right\}$. Then the following two assertions are equivalent:

1) $\left\{X_{i}: i \in I\right\}$ is an admissible family of sets;

2) for any injective finite sequence $\left\{i_{0}, i_{1}, i_{2}, \ldots, i_{k}\right\} \subset I$, we have

$$
\operatorname{card}\left(X_{i_{0}} \backslash\left(X_{i_{1}} \cup X_{i_{2}} \cup \cdots \cup X_{i_{k}}\right)\right) \geq \omega .
$$

We omit an easy verification of the equivalence of 1) and 2).

Theorem 3. Let $\left\{X_{i}: i \in I\right\}$ be a countable point-finite admissible family of sets such that

$$
(\forall i \in I)\left(\operatorname{card}\left(X_{i}\right)=\omega\right) .
$$

Then there exist mutually coherent enumerations of all members of $\left\{X_{i}: i \in I\right\}$.

Proof. We can assume, without loss of generality, that all $X_{i}(i \in I)$ are subsets of $\omega$ and

$$
\cup\left\{X_{i}: i \in I\right\}=\omega .
$$

Let $\left\{Y_{n}: n \in \omega\right\}$ be a countable family of sets such that:

(a) each set $Y_{n}(n \in \omega)$ coincides with some set $X_{i}$ where $i=i(n)$;

(b) for each index $i \in I$, we have $\operatorname{card}\left(\left\{n \in \omega: Y_{n}=X_{i}\right\}\right)=\omega$.

We are going to define by recursion a sequence of partial functions

$$
f_{n}: \omega \rightarrow \omega \backslash\{0\}(n \in \omega \backslash\{0\})
$$

such that

$$
f_{1} \subset f_{2} \subset \cdots \subset f_{n} \subset \cdots
$$

and $\operatorname{card}\left(\operatorname{dom}\left(f_{n}\right)\right)<\omega$ for any $n \in \omega \backslash\{0\}$. Suppose that the functions $f_{1}, f_{2}, \ldots, f_{n}$ have already been defined and consider the sets $Y_{n+1}, \operatorname{dom}\left(f_{n}\right)$, $\operatorname{ran}\left(f_{n}\right)$. Denote

$$
J=\left\{i \in I: X_{i} \neq Y_{n+1}, X_{i} \cap \operatorname{dom}\left(f_{n}\right) \neq \varnothing\right\} .
$$

Since the original family $\left\{X_{i}: i \in I\right\}$ is point-finite and the set $\operatorname{dom}\left(f_{n}\right)$ is finite, we claim that the set $J$ is finite, too. Consequently,

$$
Y_{n+1} \backslash\left(\cup\left\{X_{i}: i \in J\right\}\right) \neq \varnothing .
$$

Choose an element $x$ from $Y_{n+1} \backslash\left(\left\{X_{i}: i \in J\right\}\right)$ and put $f_{n+1}(x)=k$ where $k$ is the smallest element of $\omega \backslash\left(\{0\} \cup \operatorname{ran}\left(f_{n} \mid Y_{n+1}\right)\right)$. Further, let $y$ be the smallest element of $Y_{n+1} \backslash\left(\operatorname{dom}\left(f_{n}\right) \cup\{x\}\right)$. We put $f_{n+1}(y)=r$ where $r$ is some element of $\omega \backslash\left(\operatorname{ran}\left(f_{n}\right) \cup\{k\}\right)$. In this manner, the function

$$
f_{n+1}: \operatorname{dom}\left(f_{n}\right) \cup\{x, y\} \rightarrow \omega \backslash\{0\}
$$

will be determined which extends $f_{n}$. Continuing this procedure, we get the required sequence $\left\{f_{n}: n \in \omega \backslash\{0\}\right\}$ of partial functions acting from $\omega$ into $\omega \backslash\{0\}$. Finally, we define

$$
f=\cup\left\{f_{n}: n \in \omega \backslash\{0\}\right\} .
$$


It directly follows from our construction that $f$ is a function and

i) $\operatorname{dom}(f)=\cup\left\{X_{i}: i \in I\right\}=\omega$;

ii) for each $i \in I$, the restriction $f \mid X_{i}$ is a bijection between $X_{i}$ and $\omega \backslash\{0\}$.

We thus see that the function $f$ induces mutually coherent enumerations of all members of $\left\{X_{i}: i \in I\right\}$, and the proof is completed.

Remark 3. It is not difficult to show that, in Theorem 3, both assumptions on $\left\{X_{i}: i \in I\right\}$ (namely, the point-finiteness and admissibility) are essential for the validity of this theorem.

The following example is closely connected with Theorem 3 and can be regarded as an infinite version of Latin squares (in this context, cf. Example 1).

Example 2. Let $Z$ denote the set of all integers, $R^{2}=R \times R$ denote the Euclidean plane and let $Z^{2}=Z \times Z$ be the set of all those points in $R^{2}$ whose both coordinates are integers.

We shall say that a set $L \subset Z^{2}$ is a line in $Z^{2}$ if there exists a straight line $L^{\prime}$ in $R^{2}$ such that

$$
\operatorname{card}\left(L^{\prime} \cap Z^{2}\right) \geq 2, L=L^{\prime} \cap Z^{2} .
$$

It follows from this definition that every line in $Z^{2}$ contains infinitely many points.

For instance, we have two canonical countable families of horizontal and vertical lines in $Z^{2}$ :

$$
\begin{aligned}
& L_{k}=\{(m, k): m \in Z\} \quad(k \in Z), \\
& P_{k}=\{(k, m): m \in Z\}(k \in Z) .
\end{aligned}
$$

Suppose now that we are given a countable family $\left\{Q_{i}: i \in I\right\}$ of lines in $Z^{2}$ which satisfies the assumptions of Theorem 3. Actually, it suffices to suppose only that $\left\{Q_{i}: i \in I\right\}$ is point-finite (since, as easy to show, any family of lines in $Z^{2}$ is admissible). Then, by virtue of Theorem 3, we can assert that there exist mutually coherent enumerations of all members of $\left\{Q_{i}: i \in I\right\}$.

In particular, if

$$
\left\{Q_{i}: i \in I\right\}=\left\{L_{k}: k \in Z\right\} \cup\left\{P_{k}: k \in Z\right\},
$$

then our $\left\{Q_{i}: i \in I\right\}$ is point-finite and, applying the same theorem, we conclude that there exists a function

$$
f: Z^{2} \rightarrow \omega \backslash\{0\}
$$

such that, for any $k \in Z$, the restriction $f \mid L_{k}$ is a bijection from $L_{k}$ onto $\omega \backslash\{0\}$ and the restriction $f \mid P_{k}$ is a bijection from $P_{k}$ onto $\omega \backslash\{0\}$.

Note that analogous examples can be given for the case of the $n$-dimensional Euclidean space $R^{n}(n \geq 3)$ and for the subset $Z^{n}$ of $R^{n}$ consisting of those points whose all coordinates are integers.

Example 3. We recall that a family $\left\{X_{i}: i \in I\right\}$ of infinite sets is almost disjoint if $\operatorname{card}\left(X_{i} \cap X_{j}\right)<\omega$ for any $i \in I, j \in I, i \neq j$. The notion of an almost disjoint family of sets was first introduced by Sierpiński who showed 
that there exists an almost disjoint family $\left\{X_{i}: i \in I\right\}$ of subsets of $\omega$ with $\operatorname{card}(I)=2^{\omega}$ (see, e.g., [6], [7]). This fact found numerous applications in mathematical logic, axiomatic set theory, infinite combinatorics, the theory of Banach spaces, etc. It readily follows from the definition that any almost disjoint family of subsets of $\omega$ is admissible. Thus, in view of Theorem 3, we can assert that if $\left\{X_{i}: i \in I\right\}$ is a point-finite almost disjoint family of subsets of $\omega$, then there exist mutually coherent enumerations of the members of $\left\{X_{i}: i \in I\right\}$. Note that the family of all lines in $Z^{2}$ (see Example 2) forms an almost disjoint family of sets. Moreover, the intersection of any two distinct lines in $Z^{2}$ is always a one-element set (i.e., a singleton).

\section{REFERENCES}

1. M. Hall, JR., Combinatorial theory. Blaisdell Publishing Co. Ginn and Co., Waltham, Mass.-Toronto, Ont.-London, 1967.

2. K. A. Rybnikov, Introduction to combinatorial analysis. 2nd ed. (Russian) Moskov. Gos. Univ., Moscow, 1985.

3. R. Engelking, General topology. (Translated from the Polish) Monografie Matematyczne, Tom 60. [Mathematical Monographs, Vol. 60] PWN-Polish Scientific Publishers, Warsaw, 1977.

4. R. RAdo, Axiomatic treatment of rank in infinite sets. Canadian J. Math. 1(1949), $337-343$.

5. A. Robinson, Introduction to model theory and to the metamathematics of algebra. North-Holland Publishing Co., Amsterdam, 1963.

6. Handbook of mathematical logic. Edited by Jon Barwise. Studies in Logic and the Foundations of Mathematics, Vol. 90. North-Holland Publishing Co., Amsterdam-New YorkOxford, 1977.

7. A. Kharazishvili, Elements of the combinatorial theory of infinite sets. (Russian) Tbilis. Gos. Univ., Inst. Prikl. Mat., Tbilisi, 1981.

(Received 3.09.2004)

Author's address:

I. Vekua Institute of Applied Mathematics

I. Javakhishvili Tbilisi State University

2, University St., Tbilisi 0143

Georgia

E-mail: kharaz2@yahoo.com 